\title{
APROXIMACIÓN CONCEPTUAL DE LA CARACTERÍSTICA HOLÍSTICO-HOLOGRÁFICA DE LA EVALUACIÓN DOCENTE*
}

\author{
La cultura de la evaluación escolar, en la perspectiva transformacional \\ del centro escolar del siglo XXI, requiere una evaluación de los maestros \\ que interrelacione la condición autopoética y práxica del ejercicio profesional \\ de la docencia con el desarrollo institucional integrado.
}

Elías Tapiero Vásquez

\section{Resumen}

El presente artículo es un intento de conceptualización, desde el pensamiento complejo, sobre la evaluación docente en correspondencia con un concepto de evaluación más interrelacionado y global en educación, para potenciar la autopoiésis del centro escolar como fundamento de la autorregulación institucional, y, por tanto, de la autonomía escolar. En tal sentido, la característica holística-holográfica de la evaluación docente se circunscribe en el Modelo de desarrollo institucional integrado, MDII, como resultado de los avances teórico-prácticos del Proyecto cofinanciado por Colciencias, "Un estudio de caso de gestión institucional integrada en educación básica, referente para la formación del profesional de la docencia en la Universidad de la Amazonia", que dirige
el autor.

Palabras clave: Modelo de desarrollo institucional integrado, característica holístico-holográfica, autopoiésis social, pensamiento complejo, evaluación autorregulativa.

\section{Abstract}

With this article, the author tries to conceptualize, from the complex though, the teaching assessment according to a more interrelated and global concept of evaluation in Education in order to promote the autopoiesis at the

\footnotetext{
* Docente-investigador de la Universidad de la Amazonia (Florencia, Caquetá). E-mait: zyx77@telecom.com.co

** El contenido del presente artículo fue esbozado por el autor en el panel. "La evaluación docente", en el Encuentro Internacional sobre Nacional, Centro de Convenciones Alfias en Evaluación Educativa: Consecuencias para la Educación. Bogotá: Universidad Pedagógica Nacional, Centro de Convenciones Alfonso López Pumarejo, Universidad Nacional de Colombia, mayo de 2004.

Este texto fue recibido el día 19 de octubre de 2004 y aprobado el día 3 de diciembre de 2004.
} 
school, as a foundation of institutional self-regulation and school autonomy, as well. Thus, the holistic characteristic of teaching assessment is confined to the Integrated Institutional Development Model (IIDM). It appears as a result of the theoretical and practical advances cofinanced by Colciencias, which is a case Study of Institutional Management Integrated in Basic Education, Regarding the Professional Training of the Teaching Process at the Amazonia University, and directed by the author of this article.

Key words: Integrated institutional development model (IIDM), holistic-holographic characteristic, social autopoiesis, complex thought, evaluation autorregulative.

\section{INTRODUCCIÓN}

La evaluación docente se redimensiona al asumir la gestión pedagógico-administrativa del centro escolar como un todo, lo cual es posible por el Modelo de Desarrollo Institucional integrado, MDII, cuya relación entorno/sistema deriva la connotación holístico-holográfica que conlleva a la conciencia de la reestructuración sistémica del centro escolar.

EI MDII se fundamenta en la organización sistémica del centro escolar en atención a las exigencias que plantea el mundo globalizado, y propende hacia nuevas estructuras de gestión administrativo-pedagógica, en correspondencia con el ejercicio profesional de la docencia, el papel cultural del centro escolar y la formación integral de los estudiantes. Lo holístico deviene del entorno del Modelo, y lo holográfico lo define la concreción de una red de relaciones internas del Modelo, a partir de la reestructuración sistémica o de procesos en red, para transformar lo irrelevante, paradójico, anómalo, unilineal, atomizado e insular de la gestión escolar, producto del desarrollo institucional integrado por la comunidad educativa, lo cual equivale a reducir el exceso de trabajo, generar sincronización entre los desarrollos del aula con la proyección social y producir selectividad frente a las presiones coyunturalistas y heterorregulantes, como producto de la asertividad en la micropolítica escolar, es decir, de gestionar con calidad la autonomía escolar. En consecuencia, la reestructuración sistémica en la relación holísticoholográfica confronta el conformismo y la "colaboración" cómoda, complaciente, artificiosa y formalista, propios de la hiperformalización ${ }^{1}$, capaz de resemantizar el sentido tradicional y prevaleciente de modernización escolar forjada en procesos de heterorregulación extrema.

A continuación se presenta la característica holística del MDII para identificar dicha connotación en la evaluación docente.

\section{LA CARACTERÍSTICA HOLÍSTICA DE LA EVALUACIÓN DOCENTE}

Lo holístico proviene del nuevo paradigma de la ciencia donde la relación entre las partes y el conjunto es simétrica, en tanto las partes se entienden por completo a través de la dinámica del conjunto ${ }^{2}$; de alli que lo holístico se relaciona con lo global, lo cósmico.

' La hiperformalización corresponde a los procesos de mejoramiento institucional en apariencia, como resultado de una visión acritica y extremadamente formal del desarrollo institucional por las contradicciones entre lo escrito en los documentos y la conservación de los factores de obsolescencia por modificar. Es el resultado de la entropía al instaurarse en una mirada unilineal y fragmentada sobre el todo por representar el paradigma de la simplicidad, lo cual niega posibilidades de autorregulación y la consecuente creatividad e innovación en los procesos administrativo-pedagógicos, o dicho en otras palabras, la hiperformalización le impone la improbabilidad a la probabilidad de la transformación escolar.

${ }_{2}$ El pensamiento del nuevo paradigma de la ciencia asume seis criterios: la relación
Las propiedades holísticas del MDII las constituye el entorno con base en las megatendencias educativas del siglo XXI y la autonomía escolar, de manera que el entorno considera en forma crítica "la globalización" y "la sociedad civil global", al relacionarse respectivamente con la educación "antropocéntrica" y "biocéntrica".

La globalización está basada en "una red de máquinas cuyo único valor es hacer dinero para hacer dinero con la exclusión de cualquier otro valor"3, como resultado de una economía que responde al capital internacional especulativo constitu-

entre la parte y el todo; el cambio de pensamiento centrado en la función de la estructura a un pensamiento que indaga la función del proceso; la inexistencia de estructuras fundamentales e inmodificables por la identificación de la estructura que le subyace a cada proceso; la referencia de la naturaleza no excluye los seres humanos; el conocimiento como construcción carece de verdades definitivas; las verdades son descripciones aproximadas; y existe modificación radical de los métodos y los valores en los que descansa la ciencia y la tecnología. Los dos primeros criterios se refieren a la visión de la naturaleza, y los cuatro restantes se enuncian desde la epistemología. De igual manera, los cuatro primeros criterios son independientes, el quinto resulta crucial para la ciencia, y el último se relaciona con una postura cósmica, hologramática, en función del desarrollo sostenible del planeta. Capra, Fritjof. El tao de la física. Una explicación de los paralelismos entre la física moderna y el misticismo oriental. Madrid: Humanitas, 1992, pp. 373-374.

${ }^{3}$ Capra, Fritjif. Las conexiones ocultas. Implicaciones sociales, mediombientales, económicas y biológicas de una nueva visión de mundo. Barcelona: Anagrama, 2003, p. 85. 


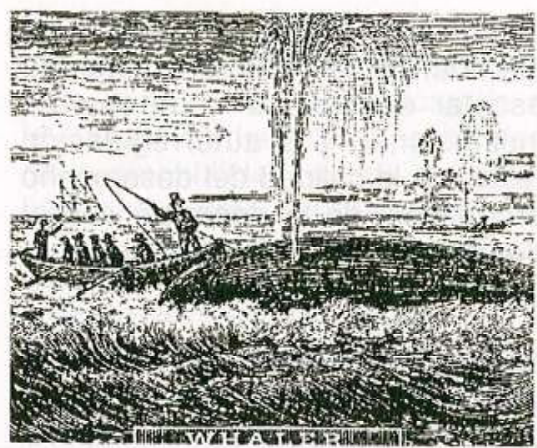

tivo de una "economía de burbuja"4, que en términos ecológicos y sociales resulta insostenible e inviable a largo plazo. La relación política temible del conocimiento en cabeza del antropocentrismo ha sido cultivada en Occidente por la universidad, las iglesias, la juridisprudencia y el Estado, con la consecuente estimulación del biocidio y el geocidio del planeta dado que propugna por una profunda y dramática fragmentación entre la vida misma -reflejada en la patología de la destrucción por la insensibilidad de los seres humanos sobre el dominio y la depredación de la naturale$\mathrm{za}^{5}$ donde la ciencia orientó sus esfuerzos en descubrir leyes, con la consecuente conversión en ideología para la dominación y el control social con base en una visión mecánica y fragmentada del universo, conocida como paradigma de la simplicidad. El biocidio (extinción diaria y acelerada de especies animales, vegetales y animales, incluida la humana a través del desplazamiento y la inanición) y el geocidio (cambio drástico de química del pla-

${ }^{4}$ Stiglitz, Joseph E. Los felices 90. La semilla de la destrucción. La década más próspera de la historia como causa de la crisis económica actual. Madrid: Taurus, 2003, pp. $63,90,279,381$.

5 Véase: Max-Neef, M. Sociedad, valores y docencia. Primer Congreso Internacional de Docencia Universitaria: Educación Superior: una reflexión necesaria. San Juan de Pasto, Universidad de Nariño. Memorias en $C D$, Conferencia, feb. 26-28, 2004, y Transdisciplina para pasar del saber al comprender, En: Debates, No. 36. Medellín: Universidad de Antioquia, 2003, No. 36. sep. dic. 2003. neta por la afectación de la atmósfera, la hidrosfera y la geosfera) representan la explotación irracional del planeta, que, estimulada por el síndrome del consumismo y la ausencia de parámetros de una sociedad incluyente, aceleró el acoso, el dominio y la depredación producidos por el desmantelamiento del complejo y asombroso programa desarrollado por la naturaleza a través de miles de millones de años de evolución, incrementando el impacto destructor de la biosfera.

Por su parte, "la sociedad civil global" emergió de la Coalición de Seattle (1999) y el Foro Social Mundial en Porto Alegre (2001) para confrontar la "globalización polarizadora"6 mediante los valores de la dignidad humana, la ética de la sostenibilidad y una postura ecológica, integrada y coherente del mundo (holística) capaz de superar reformas sectoriales tanto en administración, fiscalidad, educación y desarrollo participativo sostenible, de allí que la opción educativa es biocéntrica. El biocentrismo asume la vida en forma integral a través de una red de relaciones entre el mundo mineral, vegetal y animal, donde los humanos adquieren el compromiso de conservar el planeta, constituido éste a través de la actividad autoorganizadora, propia de los organismos con cognición ${ }^{7}$, en los parámetros del desarrollo sostenible, y que en términos educativos requiere la ecoalfabetización y el ecodiseño.

El desarrollo sostenible surge del contexto sistémico de la vida y en la comprensión autoorganizadora de los organismos con la cognición para maximizar el uso de la biomasa y minimizar la condición de residuo,

\footnotetext{
${ }^{6}$ Monereo, Manuel y Riera, Miguel. (eds.), Porto Alegre. Otro mundo es posible. España: El Viejo Topo, 3a. ed., 2002, p. 260. 7 Para ahondar en el concepto del planeta como "organismo vivo", véase Capra, Teoría de la Gaia, en op. cit., 2003, pp. 28, 56$57,217$.
}

por ser éste una fuente de contaminación y degradación del medio ambiente que conlleva a la economía del desperdicio y la contaminación; el énfasis de la sostenibilidad en esta cultura radica en crear comunidades sostenibles por la capacidad desarrollada de satisfacer las necesidades sin comprometer las oportunidades de las generaciones venideras al utilizar procesos ecosistémicos y dinámicos de coevolución, aprendidos de la misma naturaleza. La ecoalfabetización se refiere al tipo de alfabetización ecológica requerida por el desarrollo sostenible, e implica una pedagogía centrada en la comprensión de la vida por ser una experiencia de aprendizaje en el mundo real que supera la separación del hombre con la naturaleza y restablece un sentido de identidad con ella, lo cual requiere planes de estudios para atender las realidades básicas de la vida (los residuos de una especie son alimentos de otra, la materia circula incesantemente por la trama de la vida, la vida es un concepto complejo que se interrelaciona con la naturaleza inorgánica y la cognición, etc.). La ecoalfabetización implica una educación para la sostenibilidad, $y$ se basa en el conocimiento que produce el ecodiseño, el cual consiste en aprender los complejos modelos que por millones de años ha desarrollado la vida a partir de los microorganismos y los respectivos ecosistemas naturales para que una comunidad humana sostenible diseñe sus formas de vida, de negocios, de economía de estructuras físicas y de tecnologías, sin interferencia con la capacidad innata de la naturaleza para sustentar la vida. La tendencia biocéntrica de la educación puede ser corroborada en instituciones, como: Center for Ecoliteracy of Berkely (www. ecoliteracy.org), el Second Nature (www.secondnature.org) y el Schumacher College (www.gn.apc. org/ shumachercollege//8.

${ }^{8}$ Ibid., pp .290-291, 278-293. 
El entorno del MDII, constituido por las tendencias planetarias de la educación, se complementa con otro referente, no menos complejo y delicado, denominado autonomía escolar, la cual es una paradoja por el propio Estado al convertirla en fuente de esquizofrenia (enfrentó el proyecto pedagógico con el financiero ${ }^{9}$ ), lo que implica, desde la pedagogía, tomar conciencia para que la micropolítica escolar se constituya en fuente que ilumine la reestructuración sistémica institucional, en correspondencia con el ejercicio profesional de la docencia y la construcción de una mirada global e interrelacionada de la educación pública sostenida por un eje cohesionador denominado responsabilidad: responsabilidad del Estado para financiar la educación y responsabilidad de los centros escolares para la formación integral en un mundo globalizado, y para evitar así la "no reciprocidad lógica"10.

9 "...mientras la Ley General de Educación - Ley 115 de 1994 asumió la pedagogía como fuente de autonomía escolar mediante la construcción del proyecto educativo institucional para responderle a la formación integral del educando (artículo 73) con la consecuente autorregulación del plan de estudios y la introducción de nuevas perspectivas didácticas para la formación requerida (77), la Ley 715 de 2001 redujo la autonomía escolar al desarrollo de competencias en materia de gestión de recursos financieros, como expresión de la tendencia privatizadora que le subyace a las políticas neoliberales bajo la supuesta política de descentralización y de 'ahorro fiscal'. Tapiero Vásquez, Elias y López de Parra, Lillyam. "El desarrollo institucional integrado, una opción de gestión de autonomía escolar para la educación del siglo XXI". Artículo inédito por publicar en la Revista Educación y Cultura, 2005, p. 1.

10 Para Estanislao Zuleta, Elogio de la dificultad y otros ensayos, 2000:14), y la no reciprocidad lógica es el método explicativo que trata de dar cuenta de los problemas, los fracasos y los errores como responsabilidad exclusiva del adversario a quien se le aplica el esencialismo, y se deja para la excusa y la argumentación el circunstancialismo. Este tipo de lógica constituye parte de un pensamiento acrítico y unidireccional representado en el pensamiento unidireccional del paradigma de la simplicidad.
La autonomía escolar se relaciona con la ampliación de las decisiones sobre la política escolar por la comunidad educativa, y dota de libertad y responsabilidad a los centros escolares sobre la formación requerida en los nuevos tiempos, con la consecuente redefinición de las relaciones intrainstitucionales e interinstitucionales y la capacidad autorregulativa del currículo escolar en los ámbitos de la intra, inter y transdisciplinariedad ${ }^{11}$, razón por la cual la autonomía escolar se mueve en tres ámbitos mutuamente interdependientes: la identidad institucional, la iniciativa institucional y la capacidad de gestión institucional. La primera, reconoce la existencia o no de procesos integrados y coherentes del desarrollo institucional para producir alternativas; la segunda, establece la toma de decisiones para reorientar el énfasis del PEI en virtud de asumir con calidad la formación integral y, la tercera, pone en marcha, evalúa, interpreta y

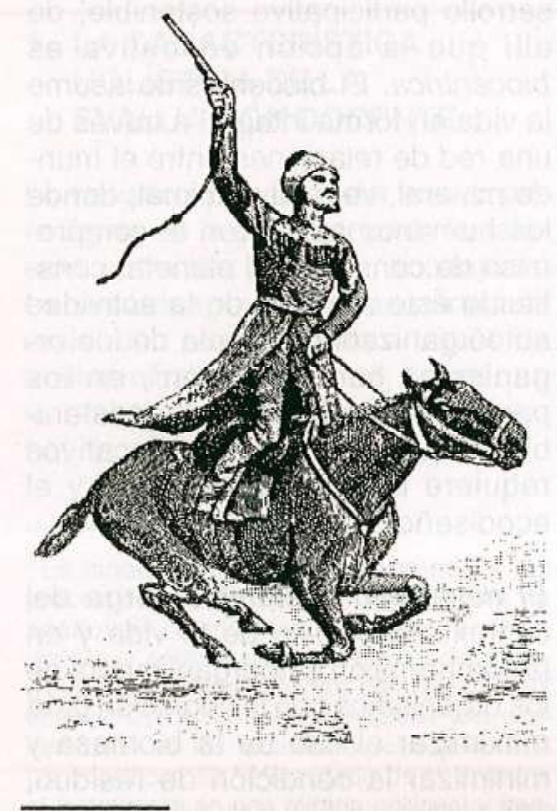

11 Dichos referentes se constituyen en ideas fuerza a la hora de definir las políticas de desarrollo institucional integrado que caracteriza el Modelo de Desarrollo Institucional Integrado, MDII, por ser constitutivas del ejercicio pleno de autonomía escolar tanto por los directivos como por los maestros y como requisito importante para superar la condición premoderna del centro escolar. redirecciona el tipo de gestión requerida; de allí que la autonomía escolar equivale a la autonomía institucional, y la autorregulación relaciona la calidad del desempeño profesional de la docencia con el desarrollo institucional integrado (Tapiero, 2001, 422-433).

La relación de las megatendencias de la educación y la autonomía escolar como entorno del MDII aporta una visión holistica y con ella prospectiva, sobre la educación del siglo XXI para liderar la incorporación de nuevas estructuras de procesos administrativo-pedagógicos con capacidad de disipar el énfasis heterorregulante que ha configurado la historia de los centros escolares. En tal sentido, la evaluación docente queda signada por una visión más universal, más global, más comprensiva del papel que le compete históricamente asumir al nuevo profesional de la docencia, otorgándole un significado especial a la conciencia y al lenguaje para que la autoevaluación, la coevaluación y la heteroevaluación direccionen nuevos rumbos del centro escolar, empoderados éstos en la producción de conocimiento e información desde la investigación, con una micropolítica que fortalezca la red de relaciones conscientemente constituida.

Dicha lógica invalida la unidireccionalidad y la fragmentación que tipifica la evaluación tradicional y la evaluación por logros, al establecer articulaciones autorreguladas del desempeño profesional de la docencia en virtud del desarrollo institucional sistémico en conjunción con el campo de tensión constituido por las megatendencias educativas y la autonomía escolar referenciadas. Así, la superación del énfasis endogenizado y pragmático de la evaluación docente le otorga madurez a su desarrollo por potenciar la construcción de masa crítica entre los maestros y gestionar la praxis educativa en una visión global del ámbito escolar. Este referente está soportado por la Teoría de la doble 
contingencia, y es complementado con la Teoría de las estructuras disipativas, la primera, por otorgarle a la evaluación docente la posibilidad de la probabilidad, desde la improbabilidad misma, en el contexto de la Teoría de la complejidad que fundamenta la Teoría de los sistemas sociales de Niklas Luhmann ${ }^{12}$ y, la segunda, dota de sentido la condición de organismo vivo e inteligente del centro escolar como posibilidad disipativa de los procesos heterorregulantes mencionados en la concreción de autopoiésis social en estrecha relación con la praxis educativa.

Planteada la característica holística de la evaluación docente, a continuación se presenta su condición holográfica.

\section{LACARACTERÍSTICA HOLOGRÁFICA DE LA EVALUACIÓNDOCENTE}

Lo holográfico es parte constitutiva del pensamiento complejo en concordancia con la dinámica holística presentada; supera la racionalidad aristotélica del "todo como la suma de las partes" por asumir que en las partes se refleja el todo; precisa que las partes no son el todo ni que el todo equivale a las partes, porque el todo y las partes operan como caras de una misma moneda; dicho de otra manera, el todo y las partes son polos complementarios.

La característica holográfica retoma la especificidad organizacional del sistema que representa el MDII a través de la red dinámica y creativa de relaciones administrativo-pedagógicas por efectos de la autorregulación institucional proveniente de la autopoiésis social ${ }^{13}$. Dicha red está concebida a partir de los presupues-

12 Véase Luhmann, N. Sistemas sociales. Lineamientos para una teoría general. Barcelona: Anthropos, Universidad Iberoamericana, Centro Editorial Javerino [sic], 1998, pp. 113-139.

${ }^{13}$ La autopoiésis es un concepto proveniente de la microbiología aportado por Humberto Maturana y Francisco Varela, precisado tos teóricos provenientes tanto de la Teoría de las estructuras disipativas de Illya Prigogine, como de la ciencia crítica de la educación en los términos de Wilfred Carr y Sthepem Kemmis, en la condición sistémica de la Teoría de los Sistemas Sociales de Niklas Luhmann. La especificidad epistemológica se deriva de la autopoiésis social, el sistema cerrado emergente y la praxis educativa, cuya interrelación a construir se comprende desde un tipo de pedagogía nutrida por la autopoiésis social y la praxis educativa, a fin de reconceptualizar la pedagogía sistémica tradicional construida con aportes de Bertallanffy y las posturas del estructural-funcionalismo de Parson, para reconfigurar los desarrollos alternativos del neoinstrumentalismo que caracteriza los giros recientes de la reforma educativa de corte neoliberal que sostiene la tendencia antropocéntrica de la educación. Desde el ámbito metodológico, el aspecto holográfico del MDII pone en marcha la relación en red del mundo escolar a partir de la planeación institucional integrada, PII, y la investigaciónacción, I-A, para construir un PEI proceso a través de los ejes político-administrativo, pedagógico-didáctico y comunicacional.

por la Teoria de las estructuras disipativas de llya Prigogine y sus colaboradores, que permite comprender los mecanismos de la dinámica de la estructura de los procesos de la célula basados en la simultaneidad de la conservación de equilibrio en la estructura organizacional y la producción de la emergencia de nuevas formas de orden y nuevas estructuras de procesos a través de la emergencia del "punto de bifurcación" que permite comprender cómo un sistema está dotado de la capacidad de permanecer en un estado alejado de equilibrio que conserva, a la vez, la estabilidad. La autopoiésis aplicada a la comprensión de los procesos de cambio organizacional se ha denominado autopoiésis social, y para tal fin requiere del lenguaje, la conciencia y la cultura, lo cual produce para las organizaciones la caracteristica de organismo vivo para comprender hasta qué punto y en qué formas están vivas las organizaciones humanas, de manera que se pueda plantear el cambio organizativo desde una nueva luz. Capra, op. cit., 2003, pp. 136-137.
La PII del centro escolar, por ser holográfica, interrelaciona y cohesiona la interinstitucionalidad (relaciones entre instituciones del orden educativo, cultural, económico, social y político a nivel local, regional, nacional e internacional) y la intrainstitucionalidad (coherencia de la gestión interna del centro escolar basada en la interrelación entre los desarrollos del aula con la proyección social), expresadas en un currículo que hace fluir en forma consciente las particularidades de la intradisciplinariedad (desarrollo endógeno de las áreas del plan de estudios), la interdisciplinariedad (desarrollos entre algunas áreas del plan de estudios) y la transdisciplinariedad (estudio y resolución de problemas del entorno escolar con la mediación de las áreas). La PII articula las decisiones institucionales en forma autónoma, genera condiciones disipativas de la planeación escolar heterorregulada e hiperformalizada y aporta al énfasis del PEI con identidad y diferencia. La I-A desarrolla la figura del maestro $y$ el directivo investigador, genera la praxis y la teorización de la práctica, instaura nuevas estructuras de procesos curriculares y propugna por la gestión cultural de la institución educativa mediante la formación de actores sociales comprometidos con la construcción de región. El eje politico-administrativo es liderado por los directivos y soporta la política del desarrollo institucional integrado con la mediación de los órganos de dirección del gobierno escolar para concretar avances sobre la autonomía escolar. El eje pedagógico-didáctico es dinamizadó por los maestros, y construye la interrelación y la coherencia entre el aula y la proyección social del centro escolar. El eje comunicacional potencia la autorreferencia en la comunidad educativa con base en la autodescripción y la auto-observación, para concretar la autorregulación como fuente de sinergia, empoderamiento y autopoiésis para la concreción del centro escolar como organismo vivo e inteligente. La condición de organismo vivo es 
posible configurarla a través de la neguentropía por instalarse ésta en la comprensión crítica de los procesos desarticulados y atomizados de la institución educativa al configurar un sistema cerrado emergente; de allí que se entienda que una reestructuración institucional se concibe sobre la base de producir alternativas para pasar de la desarticulación y la atomización a la interconexión y la interrelación de redes de procesos, para sostener la nueva dinámica del desarrollo institucional integrado.

Esta estructura sistémica de procesos en red, producto de la característica holográfica del MDII, le aporta el principio autorregulativo a la evaluación escolar, de manera que la evaluación autorregulativa circunscribe la particularidad de los resultados en fuente de preguntas para superar su condición unilineal, fragmentada, utilitarista y pragmática a través de la interiorización y el consecuente desarrollo de competencias en la auto-descripción y la auto-crítica, que diferencia tanto el papel de cada uno de los diversos actores involucrados de la comunidad educativa, como el tipo de interrelación que instaura en los ámbitos administrativo-pedagógicos.

La interiorización es un proceso de toma de conciencia que conlleva a cada actor a definir el grado de responsabilidad que tiene frente al tipo de resultados; una vez organizada dicha reflexión, se intercambia con los otros actores comprometidos de la comunidad educativa para concertar esfuerzos en la dirección que requiere la asunción de la visión establecida ${ }^{14}$. Por tal razón, se puede afirmar que la evaluación docente autorregulativa redimensiona el papel de la formación en democra-

\footnotetext{
${ }^{14}$ La asunción de la visión del centro escolar implica una dinámica autorreguladora por los actores del centro escolar en forma interrelacionada y coherente y requiere diferenciar la postura personal de los principios institucionales. La primera, basada en los pareceres y los voluntarismos, activa los prejuicios que usualmente conllevan a ser
}

cia en el centro escolar, para transitar del "respaldo" como sinónimo de participación, a la "participación propositiva" por la comunidad educativa y, en tal sentido, potencia la micropolítica escolar al servicio de la autonomía escolar, da cuenta de la especificidad de la articulación en red que caracteriza tanto lo sistémico como lo holográfico y aporta a la concreción de la condición holística del MDII.

A continuación, y a manera de ejemplificación, desde la especificidad del rendimiento escolar, se presentan posibles interrogantes que emergen del proceso de interiorización por los actores comprometidos en virtud de la evaluación escolar autorregulativa ${ }^{15}$ :

a) El maestro: ¿Cómo enseñé? ¿Qué fundamentación teórica me ayudó a orientar el proceso de conceptualización y construcción metodológica que asumí en el aula? ¿Qué metodología utilicé? ¿Las actividades y orientaciones del proceso de enseñanza estuvieron encaminadas a repetir conceptos o a generar nuevos procesos de pensamiento? ¿Conllevé a los estudiantes a resolver dificultades de aprendizaje? ¿Los estudiantes actúan motivados o presionados en el

severas con las personas, con los contradictores (principio de no reciprocidad lógica) pero débiles y huidizos frente a la resolución de problemas, y por tanto extremadamente ingeniosos con las justificaciones para evadir responsabilidades, de allí que constituye un terreno estéril para la autocrítica y la autoevaluación. La segunda postura conlleva a facilitar acuerdos para asumir los retos con responsabilidad, propia de los ambientes que estimulan la autorregulación, la flexibilidad, y por tanto la autoevaluación y la coevaluación para la toma de decisiones.

15 Tapiero V., Elías y López de P., Lillyam. "La evaluación del aprendizaje escolar: elemento constitutivo del desarrollo institucional integrado". Documento interno de Trabajo en el Seminario Taller Permanente, del proyecto". Un estudio de caso de gestión institucional integrada en educación básica, referente para la formación del profesional de la docencia en la Universidad de la Amazonia", 2004, p. 1 proceso de aprendizaje? ¿Cómo se introdujo la experiencia del estudiante y de su entorno en su aprendizaje? ¿Cómo resuelvo los problemas de aprendizaje escolar conjuntamente con mis colegas? ¿De qué manera contribuyo para que los problemas del aprendizaje escolar que surgen en el aula se conviertan en objeto de análisis por el Consejo Académico a fin de redireccionar el desarrollo pedagógico de la institución? ¿El aprendizaje desarrollado promueve la formación en la nueva ciudadanía?

b) El estudiante: ¿Cómo hacer para mejorar, perfeccionar, lo aprendido? ¿Cómo me responsabilizo en forma más consciente sobre mi propio aprendizaje? ¿Cómo aprendo a interrelacionar mis aprendizajes con la vida cotidiana? ¿Cómo aprovecho mejor el entorno para enriquecer los aprendizajes de la escuela? ¿Cómo desarrollo la alegría de aprender?

c) El directivo: ¿Cómo propicio mayores niveles de autonomía y participación en los estudiantes, los profesores y la comunidad en general para instituir una cultura de la excelencia académica del centro escolar que presido a partir del mejoramiento de la evaluación escolar? ¿Cómo valorar las mejores experiencias de aula para consolidar los procesos pedagógicos y curriculares en la institución? ¿Qué seguimiento y apoyo efectúo para optimizar el aprendizaje escolar? ¿Cómo ser autoridad pedagógica, curricular y didáctica para intervenir en forma pertinente con una institución educativa centrada en alcanzar la excelencia académica?

d) El padre, la madre o acudiente: ¿Cuáles son los niveles de compromiso y participación para lograr mejores resultados académicos? ¿Cómo se logra una mejor participación en los procesos de acompañamiento en el aprendizaje escolar? ¿Cómo hacer para intervenir con mi conocimiento y experiencia para aportar a la optimización del 
aprendizaje y la organización del centro escolar?

Al retomar de manera conjunta la característica holística y holográfica de la evaluación docente, ésta se encuentra determinada por los siguientes criterios:

- Relaciona la organización administrativo-académica del centro escolar con la dinámica política y económica en términos planetarios.

- Interrelaciona la formación integral con el desarrollo institucional integrado y el desarrollo sostenible para proyectar la gestión cultural del centro escolar y la construcción de región.

口 Se nutre de objetivar la micropolítica escolar para generar autonomía escolar.

- Relaciona la parte y el todo del desarrollo y la proyección institucional.

- Actualiza el sentido de la modernización institucional a la luz de la complejidad de la sociedad del conocimiento.

Por todo lo expresado, a la característica holístico-holográfica de la evaluación docente le subyace el principio de la autopoiésis social para contribuir a redimensionar el sentido de la modernización del centro escolar en el marco de la tensión constituida entre las megatendencias educativas y la construcción de autonomía escolar, a través de un universo educativo integrado, sistémico, en red, basado en la complejidad, expresado en la autorregulación y fortalecido en procesos de objetivación, capaz de afectar la historia heterorregulada e hiperformalizada del centro escolar.

La característica holístico-hologramática de la evaluación docente es un pre-texto para pensar un nuevo profesional de la docencia en la multiplicidad de los impactos que produce el campo de tensión cons tituido entre el paradigma de la simplicidad y el paradigma de la complejidad, en el mundo escolar, en congruencia con las preguntas sobre la educación y la pedagogía del siglo XXI.

\section{BIBLIOGRAFÍA}

CARR, Wilfred. Una teoría para la educación. Hacia una investigación crítica. Barcelona: Morata, $1995.175 p$

Hacia una ciencia crítica de la educación. Barcelona: Alertes, 1990. 163 p.

CAPRA, Fritjof. Las conexiones ocultas. Implicaciones sociales, mediombientales, económicas y biológicas de una nueva visión de mundo. Barcelona: Anagrama, 2003. $389 \mathrm{p}$.

El tao de la física. Una explicación de los paralelismos entre la física moderna y el misticismo oriental. Madrid: Humanitas, 1992. 420 p.

La trama de la vida. Una nueva perspectiva de los sistemas vivos. Barcelona: Anagrama, 2002. 359 p.

Colombia, Ministerio de Educación Nacional, Ley 715 de 2001.

Colombia, Ministerio de Educación Nacional, Ley 115 de 1994.

Kemmis, Stephen y CARR, Wilfred. Teoría crítica de la enseñanza. La investigaciónacción en la formación del profesorado. Barcelona: Martínez Roca, 1988. 245 p.

Lummann, Niklas. Sistemas sociales. Lineamientos para una teoría general. Barcelona: Anthropos, Universidad Iberoamericana, Centro Editorial Javerino [sic], 1998. $445 \mathrm{p}$.

MAX-NEEF, Manfred. Sociedad, valores y do cencia. Primer Congreso Internacional de Docencia Universitaria: Educación Superior: una reflexión necesaria. San Juan de Pasto, Universidad de Nariño. Memorias en CD, Conferencia, feb., 2004.

."Transdisciplina para pasar del saber al comprender". En: Debates, No. 36 Medellín: Universidad de Antioquia, septdic. 2003, pp. 21-33.

Monereo, Manuel y Riera, Miguel (eds.) Porto Alegre. Otro mundo es posible. España: EI Viejo Topo, 2002. 287 p.

Stiguitz, Joseph. E. Los felices 90. La semilla de la destrucción. La década más próspera de la historia como causa de la crisis económica actual. Madrid: Taurus, 2003. 415 p.

TAPIero VÁsquez, Elías y López de Parra, Lillyam. "El desarrollo institucional integrado, una opción de gestión de autonomía escolar para la educación del siglo XXI". Artículo inédito para la Revista Educación y Cultura, 2005.14 p.

. "La evaluación del aprendizaje escolar: elemento constitutivo del desarrollo institucional integrado". Documento interno de Trabajo en el Seminario Taller Permanente, del proyecto "Un estudio de caso de gestión institucional integrada en educación básica, referente para la formación del profesional de la docencia en la Universidad de la Amazonia". 2004. 6 p.

TAPIERo VÁsquez, Elías. "La evaluación del desempeño de los maestros: una opción para reconfigurar". Encuentro internacional sobre políticas, investigaciones y experiencias en evaluación educativa: consecuencias para la educación. Memorias. Bogotá D.C.: Universidad Pedagógica Nacional - Icfes, 2004, pp. 66-86.

"Un estudio de caso de gestión institucional integrada en educación básica, referente para la formación del profesional de la docencia en la Universidad de la Amazonia". Florencia: Universidad de la Amazonia - Colciencias, Proyecto de Investigación, 2003. $34 \mathrm{p}$.

. "EI Desarrollo Institucional Integrado, perspectiva autorreferencial de la formación integral y la reconfiguración curricular". Cali, noviembre 6-8 de 2003. Simposio Nacional Currículo, Flexibilidad, Aprendizajes, Política Educativa y Calidad de la Formación. Instituto de Altos Estudios para la Educación y la Formación de la Universidad Santiago de Cali - Grupo Editorial Nueva Biblioteca Pedagógica, 2003, 17p.

"Estudio comparativo de las representaciones sociales y la práctica curricular de los maestros de Florencia y Manizales en autonomía escolar". Medellín: Departamento de Posgrado, Facultad de Educación, Universidad de Antioquia. Tesis doctoral, $2001.602 \mathrm{p}$.

TEZANOS DE, Aracely. Maestros artesanos intelectuales. Estudio crítico sobre su educación. Bogotá: Universidad Pedagógica Nacional, 1985. $211 \mathrm{p}$.

Zuleta, Estanislao. Elogio de la dificultad y otros ensayos, $4^{\mathrm{a}}$. ed. Cali: Feriva, 2000. $168 \mathrm{p}$. 


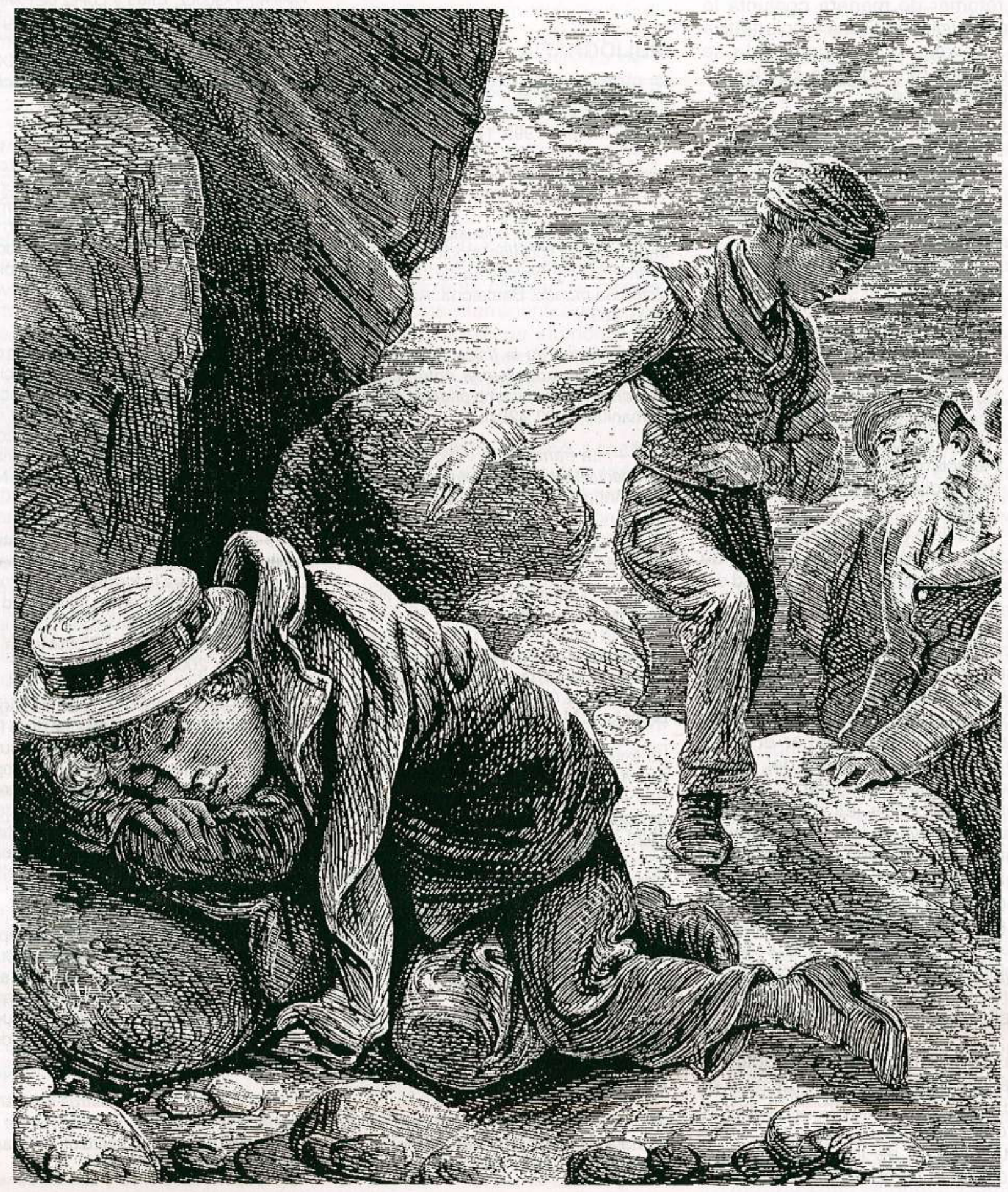

